Neuro/mages
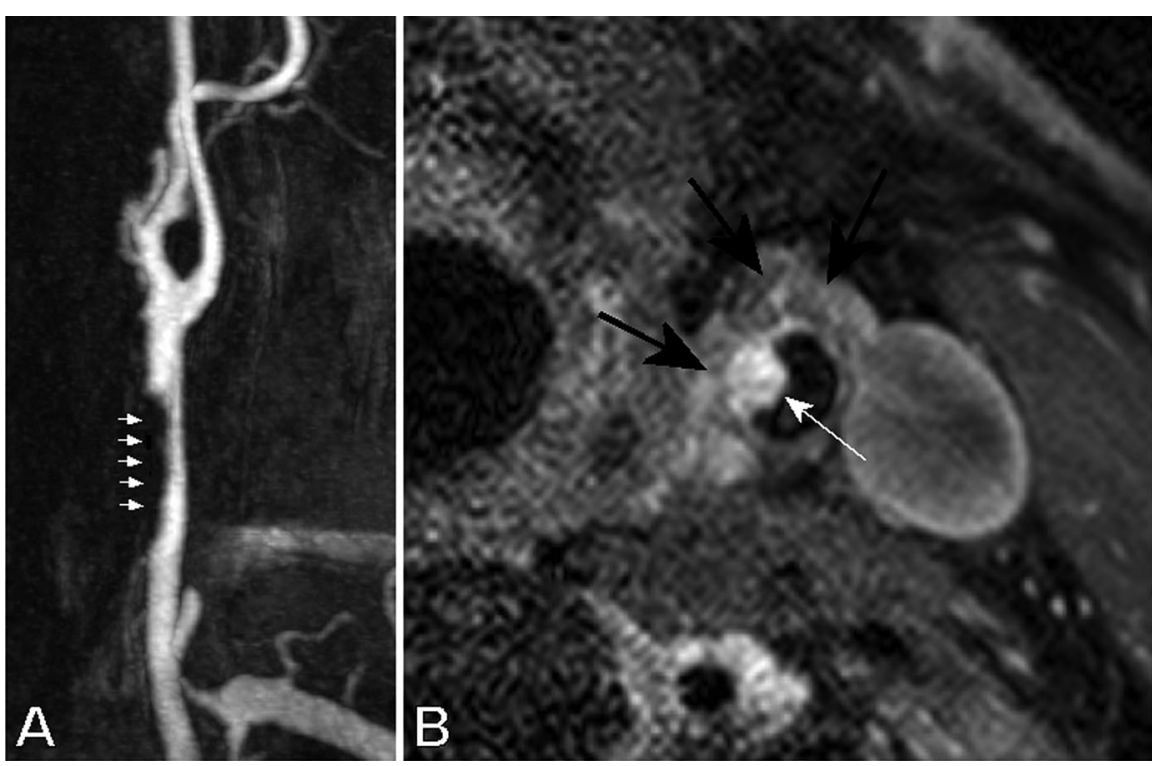

Figure. (A) Three-dimensional conventional gadolinium enhanced $M R$ angiography demonstrating an irregular stenosis of the left common artery (small white arrows). (B) Gadolinium enhanced black blood T1-weighted turbo spin echo MRI perpendicular to the common carotid artery axis showing a large plaque (black arrows) associated with an intraluminal thrombus (white arrow).

\section{High-resolution MRI of isolated intraluminal clot of the common carotid artery}

L. Boussel, MD; P. Douek, MD, PhD; and

N. Nighoghossian, $M D, P h D$, Lyon, France

A 60-year-old man developed an acute right hemiparesis with aphasia. He had hypertension and high cholesterol level. Neck ultrasound and conventional MR angiography showed a moderate

Disclosure: The authors report no conflicts of interest.

Address correspondence and reprint requests to Dr. Douek Philippe, Service de Radiologie, Hôpital Cardiologique Louis Pradel, 28 avenue du Doyen Lépine, 69677 Bron, France; e-mail: philippe.douek@creatis.univ-lyon1.fr left common carotid artery stenosis (figure, A). High-resolution MRI, performed at 1.5 Tesla using a post gadolinium T1-weighted EKG triggered black blood turbo spin echo sequence, revealed a large heterogeneous plaque with a high-intensity intraluminal thrombus (figure, B). Prothrombotic state screening was negative. Clot of common carotid artery is rare. Conventional gadolinium enhanced MR angiography may miss the clot. High-resolution MRI may better identify ${ }^{1}$ the atheromatous plaque with intraluminal thrombus.

1. Kampschulte A, Ferguson MS, Kerwin WS, et al. Differentiation of intraplaque versus juxtaluminal hemorrhage/thrombus in advanced human carotid atherosclerotic lesions by in vivo magnetic resonance imaging. Circulation 2004;110:3239-3244. 


\title{
Neurology
}

\author{
High-resolution MRI of isolated intraluminal clot of the common carotid artery \\ L. Boussel, P. Douek and N. Nighoghossian \\ Neurology 2007;68;788 \\ DOI 10.1212/01.wnl.0000256342.99552.7e
}

This information is current as of March 5, 2007

\section{Updated Information \& Services \\ Supplementary Material}

References

Citations

Subspecialty Collections

Permissions \& Licensing

Reprints including high resolution figures, can be found at:

http://n.neurology.org/content/68/10/788.full

Supplementary material can be found at:

http://n.neurology.org/content/suppl/2007/11/08/68.10.788.DC1

This article cites 1 articles, 1 of which you can access for free at: http://n.neurology.org/content/68/10/788.full\#ref-list-1

This article has been cited by 2 HighWire-hosted articles: http://n.neurology.org/content/68/10/788.full\#\#otherarticles

This article, along with others on similar topics, appears in the following collection(s):

\section{All Cerebrovascular disease/Stroke}

http://n.neurology.org/cgi/collection/all_cerebrovascular_disease_strok e

Infarction

http://n.neurology.org/cgi/collection/infarction

MRI

http://n.neurology.org/cgi/collection/mri

Information about reproducing this article in parts (figures,tables) or in its entirety can be found online at:

http://www.neurology.org/about/about_the_journal\#permissions

Information about ordering reprints can be found online:

http://n.neurology.org/subscribers/advertise

Neurology ${ }^{\circledR}$ is the official journal of the American Academy of Neurology. Published continuously since 1951, it is now a weekly with 48 issues per year. Copyright . All rights reserved. Print ISSN: 0028-3878. Online ISSN: 1526-632X.

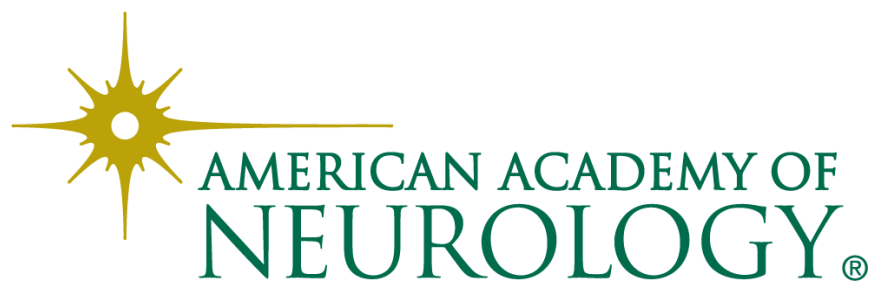

